\title{
The Immersed Boundary Method: Application to Two-Phase Immiscible Flows
}

\author{
Avihai Spizzichino ${ }^{1,2}$, Sharone Goldring ${ }^{2}$ and Yuri Feldman ${ }^{1, *}$ \\ ${ }^{1}$ Department of Mechanical Engineering, Ben-Gurion University of the Negev, \\ P.O. Box 653, Beer-Sheva 84105, Israel. \\ 2 Nonlinear Optics Group - Soreq NRC, 81800 Yavne, Israel.
}

Received 17 January 2018; Accepted (in revised version) 12 June 2018

\begin{abstract}
In this paper we present an extended formulation of the immersed boundary (IB) method that facilitates simulation of incompressible immiscible two-phase flows. In the developed formulation the pressure field and the surface tension forces associated with interface curvature are implicitly introduced in the form of distributed Lagrange multipliers. The approach provides for impermeability between both phases and exhibits accurate mass conservation without the need for additional correction procedures. Further, we present a grid independence study and extensive verification of the developed method for representative 2D two-phase flows dominated by buoyancy, shear stress, and surface tension forces.
\end{abstract}

AMS subject classifications: $76 \mathrm{~T} 10$

Key words: Immersed boundary method, two-phase immiscible flow, distributed Lagrange multiplier.

\section{Introduction}

Numerical modeling of two-phase flow is a rapidly developing field, facilitating the solution of many problems both in applied science and in engineering. Numerical simulation of two-phase flows is used for the investigation of complex phenomena in the fields of environmental and geophysical science [1,2], biomechanical engineering [3, 4], chemical processing [5] and the fabrication of optical wave guides. The simulation of two-phase flows is challenging, since it includes modeling of interactions between the different phases, tracking the boundary interface and, in some cases, resolving solidification and melting phase changes. A number of numerical techniques have been developed for modeling two-phase flows with deformable interfaces and free boundaries. Typically,

*Corresponding author. Email addresses: avihaisp@post.bgu.ac.il (A. Spizzichino), sharone@soreq.gov.il (S. Goldring), yurifeld@bgu.ac.il (Y. Feldman) 
the techniques are classified in terms of the multi-physics phenomena modeling: the flow modeling, the interface modeling, and the coupling between the two [6].

There are two basic numerical approaches that deal with free-interface two-phase flow: interface-tracking and interface-capturing. In the interface-tracking approach the interface is determined by a series of Lagrangian markers, whose location is dynamically updated throughout the numerical simulation. Front tracking (FT) $[7,8]$ and immersed boundary (IB) [9-12] methods are typical examples of the interface tracking approach. In the interface-capturing approach the interface is reconstructed from an Eulerian scalar field characterizing the flow properties. This approach includes the volume of fluid (VOF) $[8,13]$, level set (LS) $[8,14]$ and phase field (PF) methods $[8,15,16]$.

The present study focuses on the development of a novel formulation of the interfacetracking approach based on the direct forcing IB method $[17,18]$. The IB method was initially introduced by Peskin $[9,10]$ for simulation of blood dynamics in the cardiac chambers. The method is suitable for the simulation of flows in the presence of a number of immersed bodies of arbitrary geometry. Each body is determined by a set of Lagrangian points which do not necessarily coincide with the underlying Eulerian grid. In the most general case the body can be deformable and moving. In the direct forcing IB approach the kinematic non-slip constraints at all the points of the body are enforced by applying Lagrangian forces entering as sources into the Navier Stokes (NS) equations. The values of the applied forces are unknown a priori and are a part of the overall solution of the problem. In single-phase flows, the values of the Lagrangian forces are coupled with the pressure and velocity fields governed by the NS equations. In immiscible twophase flows, the simulation should also account for the surface tension forces coupling the fluid characteristics of each phase with the unknown dynamically evolving curvature of the interphase interface.

The accuracy of any two-phase numerical simulation employing the interface tracking approach depends on a precise evaluation of the Lagrangian forces on the interface between the two phases. The forces comprise the kinematic constraints for continuous values of shear stress and velocity vectors. Historically, numerical simulations relied on explicit treatment of Lagrangian forces (see e.g. Li et al. [12], Rutka and Li [19]). The surface tension forces are explicitly calculated based on the interface curvature obtained at the previous time step, while the NS equations are solved by the SIMPLE [20] algorithm. The above methodology can be easily plugged into any existing time marching solver of the NS equations based on a segregated pressure-velocity coupling, which explains its high popularity for simulation of both single- and two-phase flows [17, 18, 21,22]. However, the explicit scheme has a number of disadvantages. First, the kinematic constraints are applied to the intermediate velocity field, which has to be further projected to a divergence free subspace. As a result, a non-negligible mass leakage through the interface between the two phases typically shows up after completing the correction-projection step. To improve the accuracy and to ensure the mass conservation of the explicit direct forcing IB formulation a number of techniques have been proposed. Worthy of note are the works of Kempe et al. [23,24], who imposed substantially more accurate boundary 
conditions on the IB surface by introducing intermediate iterations. An additional important study was published by Li et al. [25], who proposed an area preservation correction scheme by correcting the interface location normally to the interface so that the area remains constant. More recently, Bao et al. [26] proposed improving volume conservation by applying a continuous velocity interpolation operator that is divergence-free, and a new force smearing operator that joins with the interpolation operator and ensures the energy conservation.

In the present study we focus on a fully coupled approach in which the momentum and the continuity equations and the Lagrangian forces, expressed as the distributed Lagrange multipliers, are fully coupled. The developed fully coupled approach offers an attractive alternative to the explicit coupling approach. The idea was originally proposed by Golowinski et al. [27], who introduced the distributed Lagrange multiplier method (DLM) for simulations of 2D flow around a moving disc [27]. An extension to this study was conducted in simulations of particulate flows [28-30] and a fluid/flexible-body interaction [31]. An additional contribution to the development of the fully coupled DLM approach was due to the work of Taira and Colonius [32], who implemented it within the framework of a projection method. The latest theoretical development of the coupled DLM approach is owing to Feldman and Gulberg [33], who extended the fully coupled approach to the linear stability analysis of pressure and thermally-driven 2D flows. The idea underlying all the implementations of the fully coupled DLM approach is that the initial system of equations is extended by including additional relationships implicitly linking the Euler flow fields and the distributed Lagrange multipliers, enforcing kinematic constraints imposed by the surfaces of immersed bodies. The main purpose of the present study is to develop and to verify extensively a novel IB formulation employing the fully coupled DLM approach for simulating two-phase immiscible flows. The developed methodology belongs to the family of FT methods and provides precise conservation of mass of both phases without the need for any additional correction procedure. In the present formulation the surface tension forces, which are proportional to the curvature of the interphase interface, are introduced as distributed Lagrange multipliers, and play the role of kinematic constraints providing impermeability of both phases. The fully pressure-velocity direct solver (FPCD), originally developed by Feldman and Gelfgat [34] for the simulation of shear- and thermally- driven confined flows, is used as a driver for presenting the capabilities of the developed methodology. The principle novelty of the developed approach stemming from the implemented fully coupled DLM formulation is twofold. First, the kinematic constraints providing impermeability of both phases are met up to the machine zero precision. Second, the developed approach provides accurate volume conservation of both phases without the need for additional correction procedures.

The rest of the paper is organized as follows. The second section presents theoretical background, including the governing equations of the impermeable two-phase flow, and implementation details of the IB and FT techniques. The third section comprises a verification study focused on simulation of four representative two-phase benchmark 
flows, followed by discussion of the obtained results. The major conclusions, as well as suggestions for future work, are then presented in the last section.

\section{Theoretical background}

Consider a viscous incompressible fluid $A$ that is immersed in a rectangular domain filled by fluid $B$, as shown in Fig.1. Each one of the fluids has a different density and viscosity represented by $\rho_{i}$ and $\mu_{i}$, respectively. The interface between the two immiscible fluids is defined by a closed boundary $-\Gamma$. The dynamics of both fluids can be described by the continuity and the NS equations:

$$
\begin{aligned}
& \nabla \cdot \boldsymbol{u}=0, \\
& \rho(I)\left(\frac{\partial \boldsymbol{u}}{\partial t}+(\boldsymbol{u} \cdot \nabla) \boldsymbol{u}\right)=-\operatorname{Re}^{-1} \nabla p+\operatorname{Re}^{-1}\left[\nabla \cdot\left(\mu(I)\left(\nabla \boldsymbol{u}+(\nabla \boldsymbol{u})^{T}\right)\right)\right] \\
& +W e^{-1} f+F^{-2} \rho(I) \vec{e}_{y} \text {, }
\end{aligned}
$$

where the non-dimensional velocity, pressure, surface tension force density and time are denoted by $\boldsymbol{u}(u, v), p, f$, and $t$, respectively. $\vec{e}_{y}$ is a unit vector in the direction which is opposite to that of the gravity force. The non-dimensional equations were obtained by using the characteristic scales $D=2 \sqrt{S / \pi}$ for the length (where $S$ is the area of fluid $A$ ), $U$ for the velocity (where $U$ is the characteristic velocity of the specific flow configuration), $t=D / U$ for the time, and $p=\mu_{\min } U / D$ for the pressure field. The mass density and the dynamic viscosity fields are scaled by the corresponding minimum values of either of the two fluids, $\rho_{\min }$ and $\mu_{\min }$.

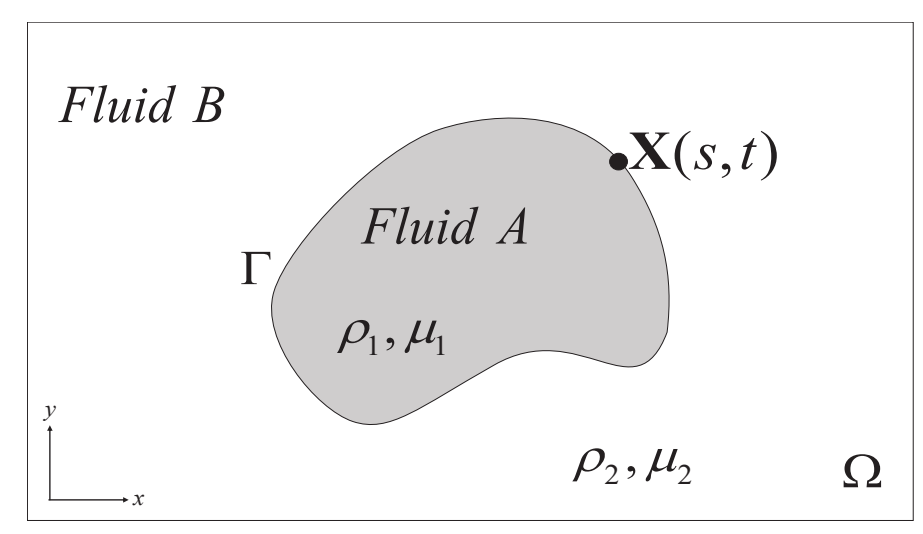

Figure 1: A schematic of a two-phase flow configuration.

In accordance with the formalism of the IB method utilized in the present study, the surface tension density $\boldsymbol{F}(s, t)$ associated with every Lagrangian point on the interface 
between the two phases is regularized to the underlying Eulerian grid by

$$
\boldsymbol{f}(\boldsymbol{x}, t)=\int_{\Gamma} \boldsymbol{F}(s, t) \delta^{2} d s, \quad \boldsymbol{F}(s, t)=\frac{\partial^{2} \boldsymbol{X}(s, t)}{\partial s^{2}},
$$

where $\boldsymbol{X}(s, t)$ determines the interface between the two phases parameterized by $0 \leq s \leq L$ ( $L$ is the total length of the interface), $\frac{\partial^{2} X(s, t)}{\partial s^{2}}$ is the local curvature of the interface and $\delta$ is the discrete Dirac delta function [35] defined as

$$
\delta(r)= \begin{cases}\frac{1}{3}\left(1+\sqrt{-3 r^{2}+1}\right) & \text { if }|r| \leq 0.5 \\ \frac{1}{6}\left(5-3|r|-\sqrt{-3(1-|r|)^{2}+1}\right) & \text { if } 0.5 \leq|r| \leq 1.5 \\ 0 & \text { otherwise }\end{cases}
$$

The dimensionless groups determining the two-phase flow under consideration are the Reynolds $(R e)$, Weber $(\mathrm{We})$ and Froude $(F r)$ numbers defined as

$$
R e=\frac{\rho_{\min } U D}{\mu_{\min }}, \quad W e=\frac{\rho_{\min } U^{2} D}{\sigma}, \quad F r=\frac{U}{\sqrt{g D}},
$$

where $g$ is the gravitational acceleration. The material properties $\rho(I)$ and $\mu(I)$ in Eq. (2.2) are a function of $I(\boldsymbol{x}, t)$, which is a scalar field, also known as an indicator function [25]. This was introduced to smear the discontinuity of material properties near the interface. Let us define a gradient of the scalar field as

$$
\nabla I(\boldsymbol{x}, t)=\int_{\Gamma} \boldsymbol{n}(\boldsymbol{X}(s, t)) \delta^{2}(\boldsymbol{x}-\boldsymbol{X}(s, t)) d s,
$$

where $\boldsymbol{n}(\boldsymbol{X}(s, t))$ is a unit vector normal to the interface between the phases. All the values of $I(x, t)$ lying in the range of $[0,1]$ are obtained by the solution of Poisson's equation:

$$
\Delta I(\boldsymbol{x}, t)=\nabla \cdot \int_{\Gamma} \boldsymbol{n}(\boldsymbol{X}(s, t)) \delta^{2}(\boldsymbol{x}-\boldsymbol{X}(s, t)) d s
$$

Following the guidelines formulated by Tryggvason et al. [7] Eq. (2.7) was solved by the SOR method. The iterations were only performed on points close to the interface (typically two grid cells in each direction), which encloses the field of influence of the discrete Dirac delta function defined by Eq. (2.4), leaving points away from the interface unchanged. The procedure made it possible to precisely keep the correct values of the density and the viscosity fields away from the interface separating between the phases. In addition, to prevent small over- and undershoots which can emerge near the interface when simulating the two-phase flows characterized by high density and viscosity ratios, a simple filtering was applied while iterating the $I(x, t)$ values. The obtained values of the indicator function $I$ were then utilized for determining the spatial variation of the density and of the dynamic viscosity fields in the vicinity of the interface between the two phases:

$$
\rho(I(\boldsymbol{x}, t))=\rho_{1}+\left(\rho_{2}-\rho_{1}\right) I(\boldsymbol{x}, t), \quad \mu(I(\boldsymbol{x}, t))=\mu_{1}+\left(\mu_{2}-\mu_{1}\right) I(\boldsymbol{x}, t) .
$$




\section{The numerical methodology}

The numerical methodology developed in the framework of the present research extends the IB formulation recently presented by Feldman and Gulberg in [33]. In this study the distributed Lagrange multiplier is formulated in terms of the local surface tension density (see Eq. (2.3)) and applied to enforce the kinematic constraint of immiscibility of both fluids on the interface between the two phases. The developed methodology is embedded into the generic incompressible NS solver, based on full pressure velocity coupling [34].

We consider 2D incompressible flow governed by the mass conservation and the NS equations, Eqs. (2.1), (2.2). Applying a second order backward finite difference scheme for time discretization, the continuity and the momentum equations can by written as

$$
\begin{aligned}
& \nabla \cdot \boldsymbol{u}^{n+1}=0 \\
& {\left[\operatorname{Re}^{-1}\left(\nabla \cdot\left(\mu(I)\left(\nabla \boldsymbol{u}+(\nabla \boldsymbol{u})^{T}\right)\right)-\nabla p\right)-\frac{3 \rho(I)}{2 \Delta t} \boldsymbol{u}\right]+W e^{-1} \int_{\Gamma} \frac{\partial^{2} \boldsymbol{X}}{\partial s^{2}}} \\
& \quad=\rho(I)\left[(\boldsymbol{u} \cdot \nabla) \boldsymbol{u}-\frac{2}{\Delta t} \boldsymbol{u}\right]^{n}+\frac{1}{2 \Delta t} \boldsymbol{u}^{n-1}-F r^{-2} \rho(I) \vec{e}_{y} .
\end{aligned}
$$

The standard staggered grid, second-order, conservative finite volume method [20] was used for the spatial discretization of all terms in Eqs. (3.1), (3.2). All the linear terms are treated implicitly, while all the non-linear terms are treated explicitly and appear on the right-hand side (RHS) of Eq. (3.2). It can be seen that as a result of surface tension forces related to the interface between the two phases, two additional unknown fields, $\left(\frac{\partial^{2} X}{\partial s^{2}}, \frac{\partial^{2} Y}{\partial s^{2}}\right)$ are introduced into the equations. In order to achieve a closed form of the extended system, two more equations are added. The additional equations relate between the interface curvature represented by the Lagrangian coordinate $X$ and the Eulerian velocity field:

$$
\left(\frac{\partial^{2} \boldsymbol{X}_{l}}{\partial s^{2}}\right)=\frac{\left(\boldsymbol{X}_{l+1}^{n+1}-2 \boldsymbol{X}_{l}^{n+1}+\boldsymbol{X}_{l-1}^{n+1}\right)}{(\Delta s)^{2}} .
$$

The coordinate $\boldsymbol{X}_{l}^{n+1}$ can further be expressed in terms of the Eulerian velocity field by the Euler integration:

$$
\boldsymbol{X}_{l}^{n+1}=\boldsymbol{X}_{l}^{n}+\Delta t \int_{\Omega} \boldsymbol{u}^{n+1} \delta^{2}\left(\boldsymbol{X}_{l}^{n}-\boldsymbol{x}\right) d x d y
$$

Performing the same procedure for $\boldsymbol{X}_{l-1}^{n+1}$ and $\boldsymbol{X}_{l+1}^{n+1}$ and further substitution into Eq. (3.3) 
yields:

$$
\begin{aligned}
& \frac{\Delta t \int_{\Omega} \boldsymbol{u}^{n+1}\left[\delta^{2}\left(\boldsymbol{X}_{l+1}^{n}-\boldsymbol{x}\right)-2 \delta^{2}\left(\boldsymbol{X}_{l}^{n}-\boldsymbol{x}\right)+\delta^{2}\left(\boldsymbol{X}_{l-1}^{n}-\boldsymbol{x}\right)\right] d x d y}{(\Delta s)^{2}}-\left(\frac{\partial^{2} \boldsymbol{X}_{l}}{\partial s^{2}}\right) \\
= & -\frac{\boldsymbol{X}_{l+1}^{n}-2 \boldsymbol{X}_{l}^{n}+\boldsymbol{X}_{l-1}^{n}}{(\Delta s)^{2}} .
\end{aligned}
$$

Eq. (3.5) is simply the kinematic constraint that provides the immiscibility of both phases. Together with Eqs. (3.1), (3.2) it forms a whole set of equations governing the dynamics of two-phase flow, which can be rewritten in a block matrix form as

$$
\left[\begin{array}{ccccc}
H_{u} & H_{u v} & -\frac{\Delta_{p}^{x}}{R e} & \frac{\delta^{2}\left(X\left(s, t^{n}\right)-x\right)}{W e} & 0 \\
H_{v u} & H_{v} & -\frac{\Delta_{p}^{y}}{R e} & 0 & \frac{\delta^{2}\left(X\left(s, t^{n}\right)-x\right)}{W e} \\
\nabla_{u}^{x} & \nabla_{v}^{y} & 0 & 0 & 0 \\
I C_{x} & 0 & 0 & -1 & 0 \\
0 & I C_{y} & 0 & 0 & -1
\end{array}\right]\left[\begin{array}{c}
u^{n+1} \\
v^{n+1} \\
p \\
\left(\frac{\partial^{2} X}{\partial s^{2}}\right) \\
\left(\frac{\partial^{2} Y}{\partial s^{2}}\right)
\end{array}\right]=\left[\begin{array}{c}
R H S_{u}^{n-1, n} \\
R H S_{v}^{n-1, n} \\
0 \\
R H S_{X}^{n-1, n} \\
R H S_{Y}^{n-1, n}
\end{array}\right],
$$

where $\nabla^{x}$ and $\nabla^{y}$ are the first derivatives with respect to the $x$ and $y$ coordinates, respectively. $H=\frac{\nabla \cdot\left(\mu(I)\left(\nabla+\nabla^{T}\right)\right)}{R e}-\frac{3 \rho(I)}{2 \Delta t} \mathbf{I}$ are modified Helmholtz operators acting on $u$ and $v$ velocity components, $\mathbf{I}$ is the identity matrix. IC is the "Interface Curvature" operator defined as

$$
I C=\frac{\Delta t \int_{\Omega}\left[\delta^{2}\left(\boldsymbol{X}_{l+1}^{n}-\boldsymbol{x}\right)-2 \delta^{2}\left(\boldsymbol{X}_{l}^{n}-\boldsymbol{x}\right)+\delta^{2}\left(\boldsymbol{X}_{l-1}^{n}-\boldsymbol{x}\right)\right] d \boldsymbol{x}}{(\Delta s)^{2}} .
$$

The computational time step of the developed methodology consists of two stages. In the first stage Poissons equation, Eq. (2.7), is solved, yielding the indicator function $I(\boldsymbol{x}, t)$, which is then utilized in Eq. (2.8) to obtain the spatial distribution of density and dynamic viscosity fields close to the interface between the two phases. In the next stage, the system of equations in Eq. (3.6) with appropriate boundary conditions ${ }^{\dagger}$ for the velocity field is solved. The solution of the system of equations is carried out by a direct multifrontal massively parallel solver (MUMPS) [36,37]. Finally, the obtained values of the velocity field are substituted into Eq. (3.4) to update the position of the interface between the two phases. After updating the interface, the Lagrangian markers are redistributed and their total number can change in order to ensure that the distance between adjacent Lagrangian points is approximately equal to the Eulerian grid size. This makes it possible to achieve a reasonable condition number of the coupled operator involved in Eq. (3.6) and to diminish round-off errors at the LU factorization stage. Note also that for the case of zero surface tension the operator in Eq. (3.6) degenerates to the Stokes

\footnotetext{
${ }^{\dagger}$ The boundary conditions are of the Dirichlet, Newman, and periodic types, each applied depending on the specific problem, as will be discussed in the next section.
} 
operator previously utilized in [34], while all other stages of the algorithm remain the same.

\section{Results and discussion}

In order to verify the developed method, four benchmark problems for incompressible two-phase flows were solved. Each flow under consideration was driven by a different mechanism, namely; buoyancy, shear, and surface tension forces. The first benchmark problem considers the flow of a circular bubble rising within a fluid of higher density due to buoyancy forces $[14,38,39]$. In general, both fluids can also have different viscosities. Under the action of buoyancy forces, the bubble accelerates while rising up until it reaches its terminal velocity. The second benchmark is the shape evolution of a drop under pure shear stress [25,40,41]. A circular drop of a certain liquid is submerged into a pure shear flow of another liquid. Both liquids have different viscosities. As a result of shear forces the submerged circular drop undergoes shape deformation. The third benchmark is known as the disc relaxation problem [25,42]. A drop of a certain fluid with an initial rosette shape is submerged in another fluid of different viscosity. As a result of surface tension forces between the two phases, the rosette-shape drop undergoes complex deformations until it eventually converges to a circular shape. Similarly to the first benchmark problem, the fourth configuration also comprises a circular bubble rising within a fluid of higher density, with the only difference being that in this case the flow is characterized by higher values of density and viscosity ratio, which eventually leads to break up of the rising bubble. The results of our simulations were compared both qualitatively and quantitatively to the data available from literature [43-45] for different Reynolds, Weber, and Froude numbers. A detailed comparison between the results is presented in the following.

\subsection{Test case 1 - Rising bubble without break up}

This benchmark focuses on the dynamics of a deformable liquid bubble submerged in a cavity filled with another fluid of higher density. This flow has been thoroughly studied both experimentally [46] and numerically [14,38,39,47,48]. The most comprehensive quantitative results are due to the review of Hysing et al. [38], which presents a comparison between the time evolutions of the circularity, the position of the center of mass and the vertical component of the velocity of the rising bubbles center of mass as defined in Eqs. (4.2)-(4.4). This data was independently obtained by three research groups, each utilizing its own numerical methodology (consult Ref. [38] for more details).

The buoyant dynamics of an initially circular rising bubble of diameter $D$ is considered. The bubble is initially placed at point $[D, D]$ within a $[2 D \times 4 D]$ rectangular domain, 


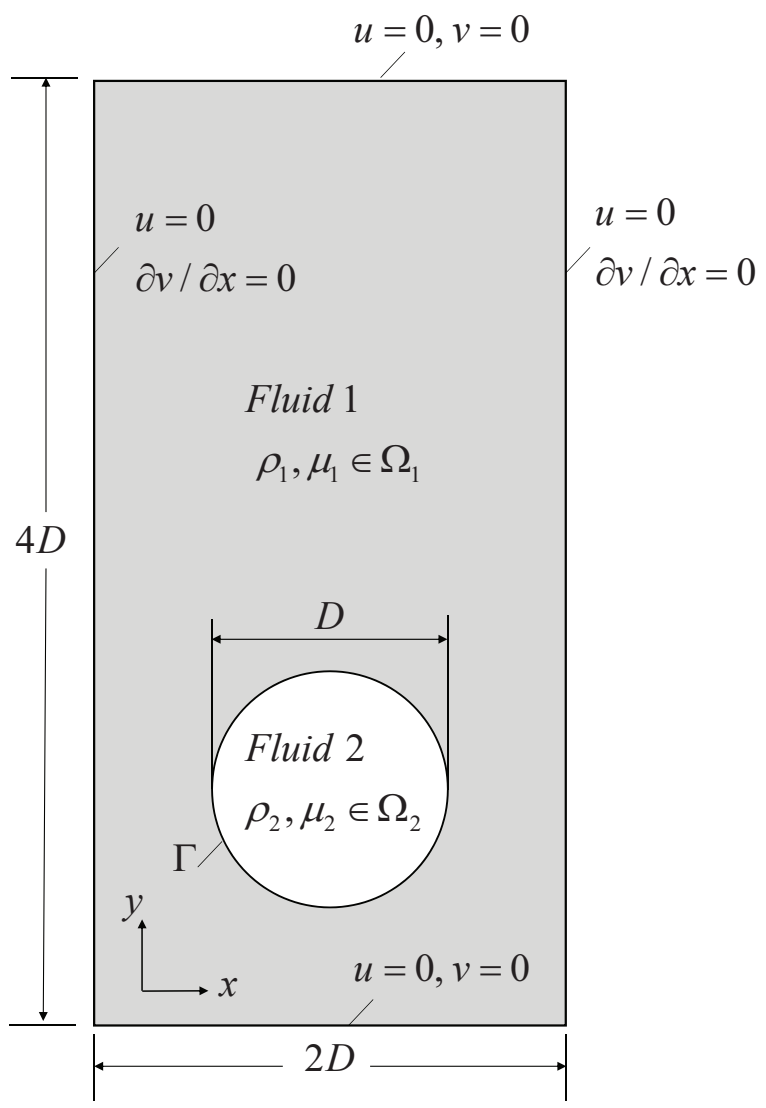

Figure 2: Initial configuration and boundary conditions of rising bubble test flow.

as shown in Fig. 2. The boundary conditions are set as:

$$
\begin{aligned}
& u(x=0, y)=u(x=2 D, y)=0, \\
& u(x, y=0)=u(x, y=4 D)=0, \\
& \frac{\partial v}{\partial x}(x=0, y)=\frac{\partial v}{\partial x}(x=2 D, y)=0, \\
& v(x, y=0)=v(x, y=4 D)=0 .
\end{aligned}
$$

In addition, a single Dirichlet point for the pressure field, $p=0$, was set in the corner of the computational domain. The values of physical properties and operating conditions governing the flow under consideration are specified in Table 1. The center of mass of the rising bubble is a local quantity given by:

$$
X_{C}=\left(X_{C}, Y_{C}\right)=\frac{\int_{\Omega_{2}} x D V}{\int_{\Omega_{2}} d V},
$$


Table 1: The values of physical properties and operating conditions.

\begin{tabular}{||cccccccccccc||}
\hline$\rho_{1}$ & $\rho_{2}$ & $\mu_{1}$ & $\mu_{2}$ & $D$ & $g$ & $\sigma$ & $\operatorname{Re}$ & $W e$ & $F r$ & $\rho_{1} / \rho_{2}$ & $\mu_{1} / \mu_{2}$ \\
\hline 1000 & 100 & 10 & 1 & 0.5 & 0.98 & 24.5 & 98.99 & 4 & 1 & 10 & 10 \\
\hline
\end{tabular}

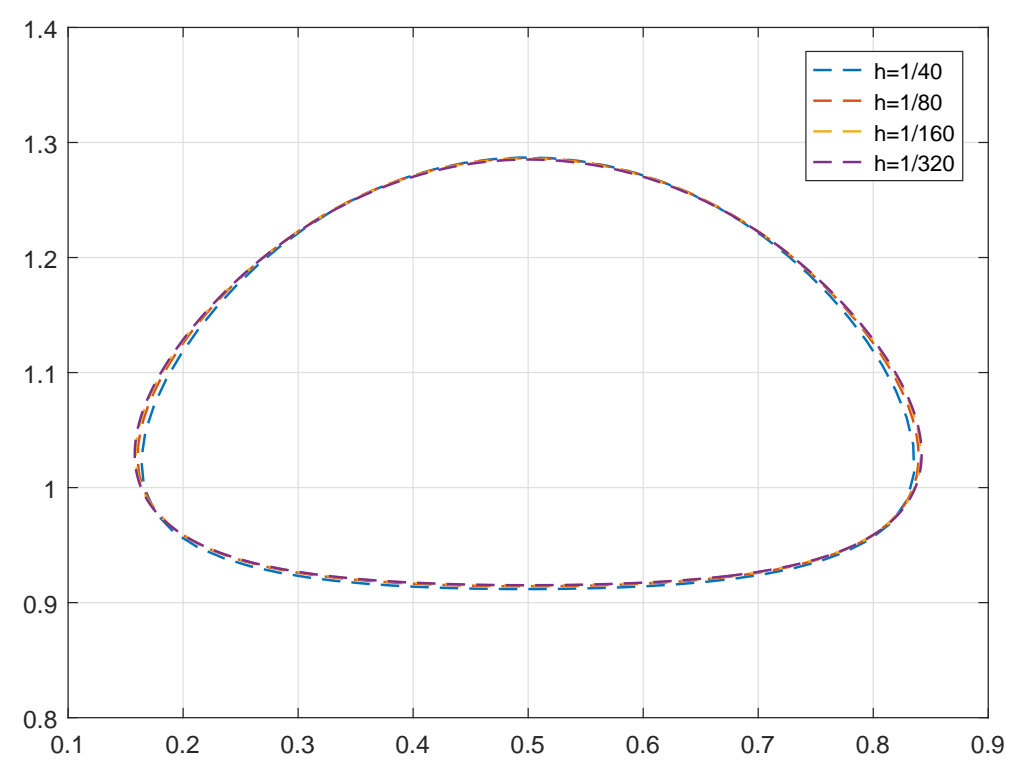

Figure 3: Bubble shapes obtained on four different grids at time $t=3$.

where $\Omega_{2}$ denotes the region occupied by the bubble. Following Wadell [49], the bubbles "degree of circularity" $\phi$ is defined in $\mathbb{R}^{2}$ as

$$
\phi=\frac{P_{a}}{P_{b}}=\frac{\pi D_{a}}{P_{b}}
$$

where $P_{a}$ is the perimeter of a circle with diameter $D_{a}$, which has an area equal to that of a bubble with perimeter $P_{b}$. The rising velocity, $U_{C}$, is defined as the mean velocity of the bubble:

$$
\boldsymbol{u}_{C}=\left(U_{C}, V_{C}\right)=\frac{\int_{\Omega_{2}} u D V}{\int_{\Omega_{2}} d V} .
$$

To prove the grid independence of the results, the simulations were performed on four different grids with a time step of $\Delta t=h / 2$, where $h$ corresponds to a grid step. A grid independence of the obtained results is favorably verified by indistinguishable differences between the final shapes of the bubble obtained at $t=3$ on the two finest grids (see Fig. 3). In addition, it can be clearly recognized that the bubble shape obtained at time $t=3$ on the finest grid is in excellent agreement with the corresponding results reported in Hysing et al. [38] (see Fig. 4). Mass conservation of the bubble is next verified by comparison 
Table 2: Mass discrepancy (\%) at $t=3$ in test case 1.

\begin{tabular}{||ccccc||}
\hline$h$ & $1 / 40$ & $1 / 80$ & $1 / 160$ & $1 / 320$ \\
\hline Mass leakage [\%] & 0.3364 & 0.0917 & 0.0560 & 0.0204 \\
\hline
\end{tabular}

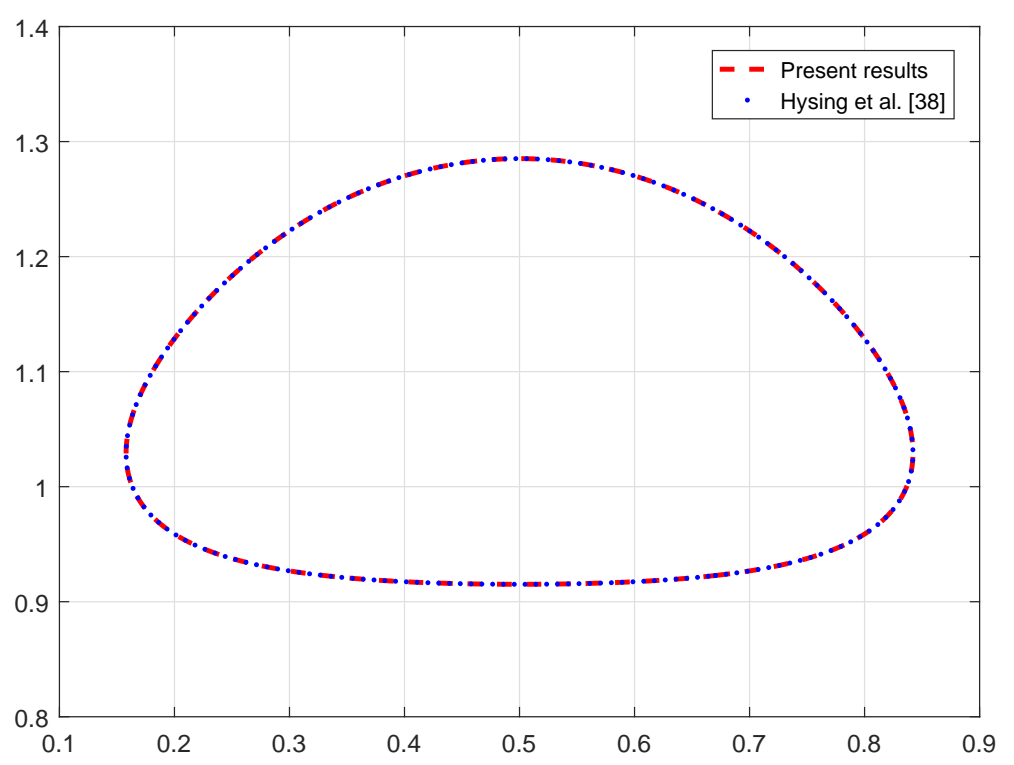

Figure 4: Bubble shapes obtained at final time $(t=3)$ : comparison between the present and the previously published results.

between the initial and the final areas of the bubble. It can be seen that for all the grids the mass lost does not exceed $0.35 \%$ of the initial mass, as summarized in Table 2. Figs. 5 , 6 and 7 compare between the present and the previously reported time evolutions of the circularity, the $Y$-axis position and the $V_{C}$ velocity component of the bubble center of mass, respectively. All the results were calculated on the grid characterized by $h=1 / 160$ with $\Delta t=1 / 16$. In addition, Table 3 presents a quantitative comparison between the corresponding instantaneous values of the global minima of circularity, global maxima of rising velocity and vertical position of center of mass at $t=3$.

It can be clearly recognized that all the presently obtained characteristics are very close to the corresponding previously obtained results for the entire range of time values. The maximal discrepancy between all the flow characteristics does not exceed $0.04 \%$. The maximal deviation between the circularities is observed around their global minima (close to the time $t=1.9$ ), characterizing the most significant deformation of the shape of a rising bubble compared to its initially circular geometry. From this point on, the circularity smoothly increases until finally attaining its asymptotic value that indicates that the bubble has reached its terminal velocity. The fact that the bubble has reached its terminal velocity is also confirmed by noting a constant slope of the time evolution 

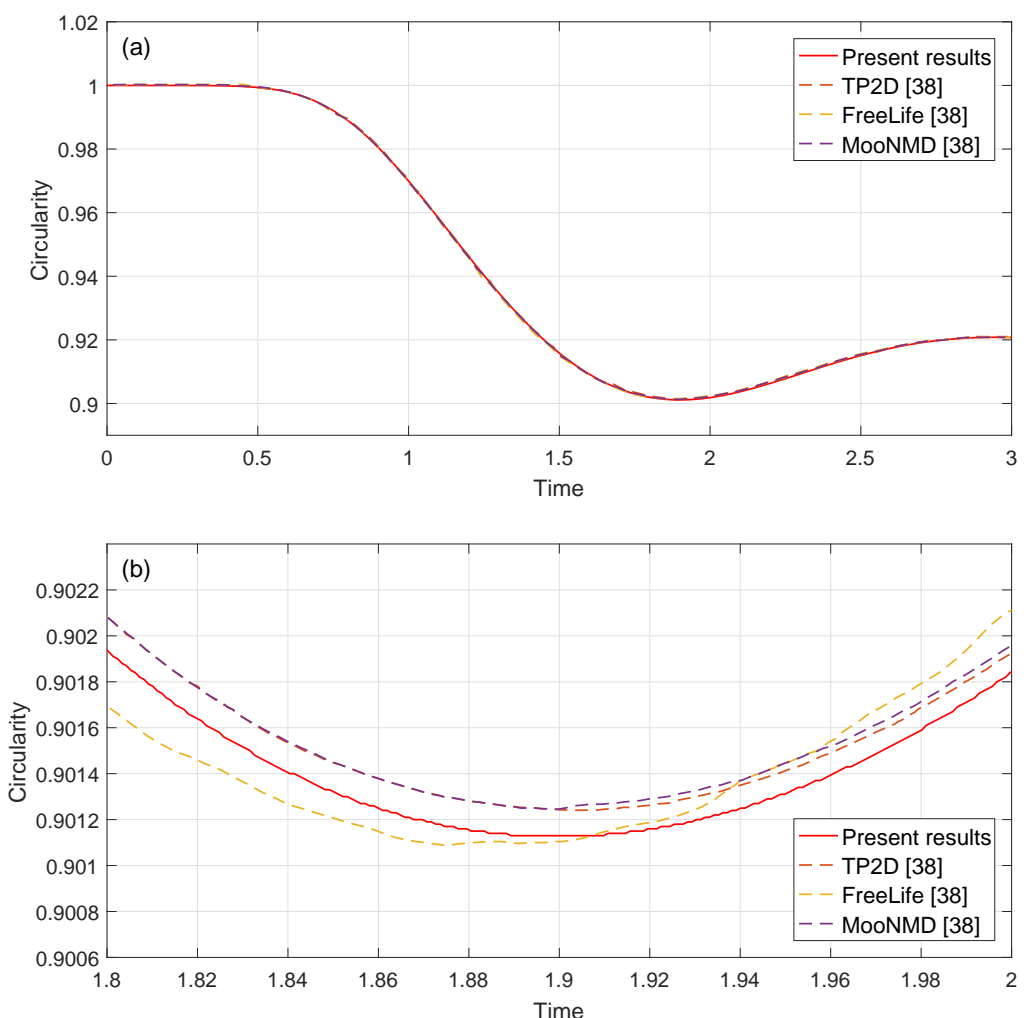

Figure 5: Time evolution of the circularity values: (a) the whole time history; (b) close-up view around the time $t=1.9$.

Table 3: . Global minima values of circularity with corresponding incident times, global maxima values of rising velocity and final vertical position of the center of mass.

\begin{tabular}{||ccccc||}
\hline & Present method & TP2D & FreeLife & MooNMD \\
\hline$\phi_{\min }$ & 0.9011 & 0.9013 & 0.9011 & 0.9013 \\
$t \mid \phi=\phi_{\min }$ & 1.900 & 1.9041 & 1.8750 & 1.900 \\
$V_{\max }$ & 0.2416 & 0.2417 & 0.2421 & 0.2417 \\
$t \mid V=V_{\max }$ & 0.9232 & 0.9213 & 0.9313 & 0.9239 \\
$Y(t=3)$ & 1.0813 & 1.0813 & 1.0799 & 1.0817 \\
\hline
\end{tabular}

of the $Y$ coordinate around time $t=2.9$ (see Fig. 6-b). It is remarkable that the value of the terminal velocity is lower than the value of the global velocity maxima observed at by about $10 \%$ (see Fig. $7-b$ ). Increasing the vertical velocity of the bubble is followed by enhanced deformation of the bubble shape, which results in a moderate deceleration of the bubble until it reaches its terminal velocity. 

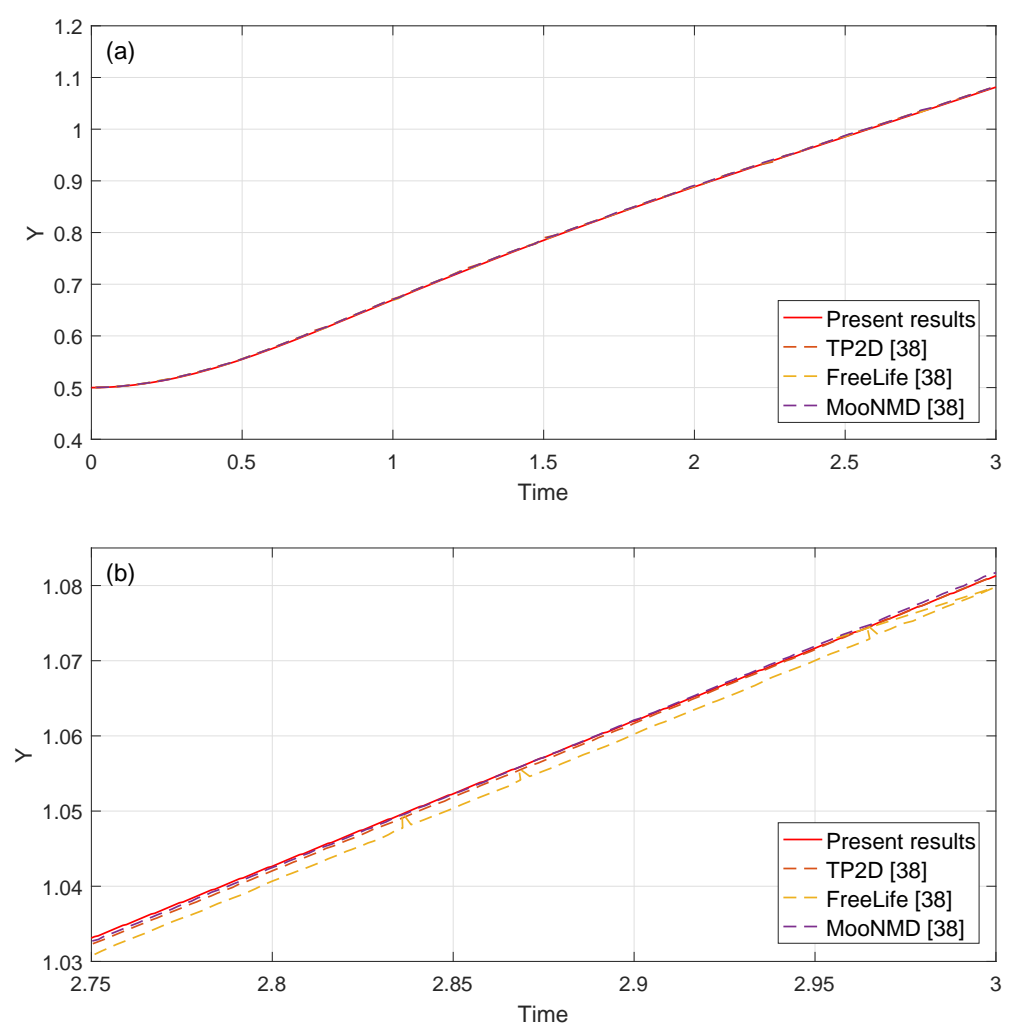

Figure 6: Time evolution of the $Y$ position of the center of mass: (a) the whole time history; (b) close-up view around the time $t=2.9$.

\subsection{Test case 2 - Drop under shear flow}

The initial state of the problem is given in Fig. 8. The circular drop submerged into fully developed Couette flow is subjected to a pure shear stress, while the gravity force is neglected. Under the action of shear stresses the flow undergoes deformation until the increasing surface-tension forces are equalized with the shear forces and the drop shape reaches equilibrium. The square box domain is of dimensions $[2 D \times 2 D]$, and the center of the submerged circular drop with diameter $D$ coincides with the geometrical center of the box (point $[D, D]$ ). Following the works of Kapil and Pozrikidis [41] and Chinyoka et al. [40] the simulations were performed for four different values of Reynolds number, $R e=1,10,50$ and 100, two values of viscosity ratio $\mu_{2} / \mu_{1}=1$ and 10 , and two values of capillary number, $C a=0.2$ and 0.4 . Note that the capillary number relates between the viscous and surface tension forces and is defined in the present study as

$$
\mathrm{Ca}=\frac{W e}{\operatorname{Re}}=\frac{U \mu_{\mathrm{min}}}{\sigma}
$$



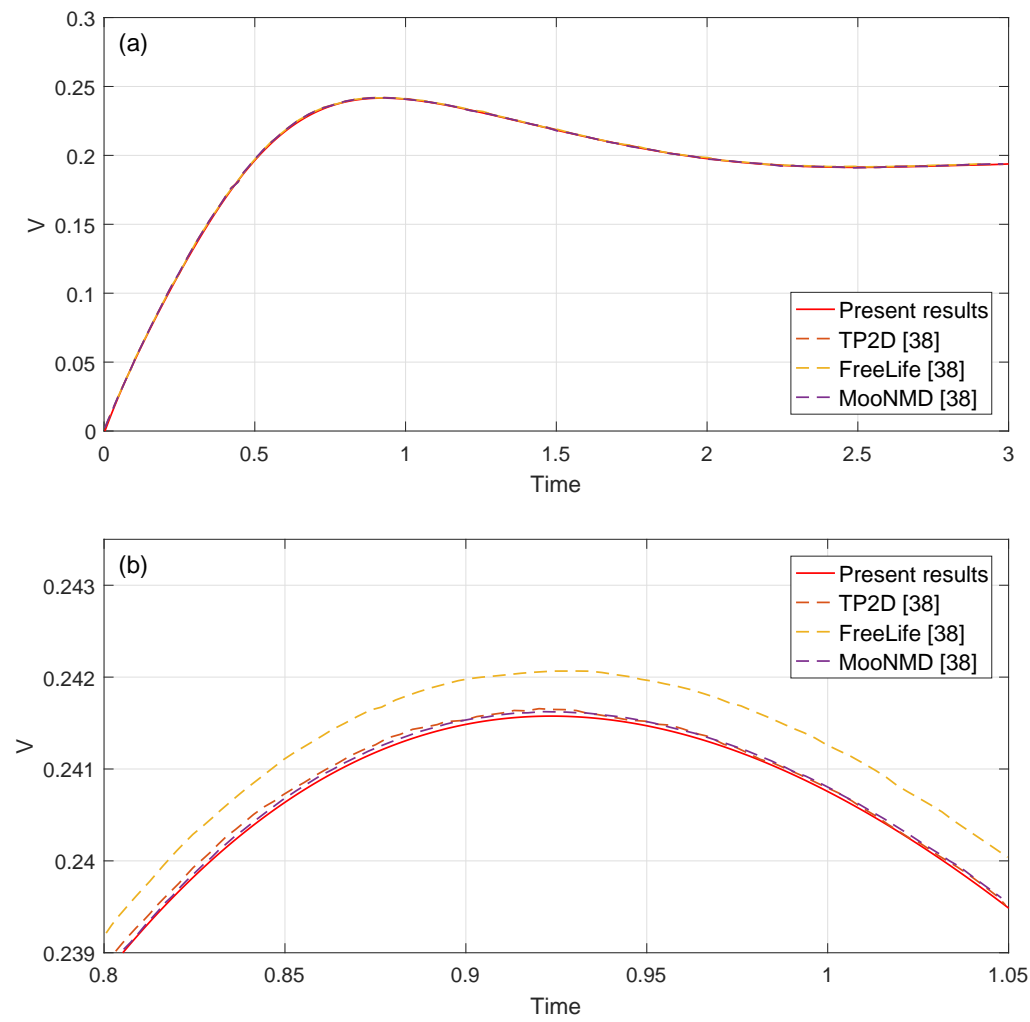

Figure 7: Time evolution of the rise velocity: (a) the whole history; (b) close-up view around the time $t=0.9$.

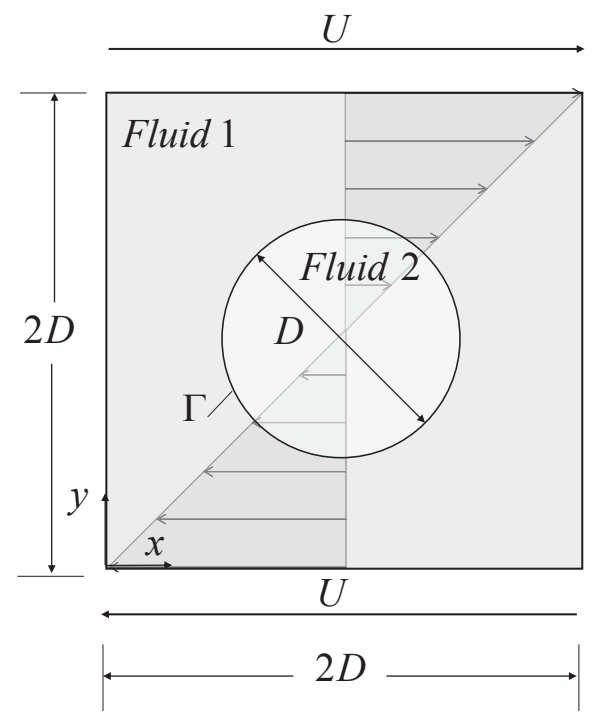

Figure 8: Initial state of the circular drop submerged into a fully developed Couette flow. 
Table 4: Mass discrepancy (\%) at $t=3$ in test case 2.

\begin{tabular}{||lllll||}
\hline & \multicolumn{2}{c}{$C a=0.2$} & \multicolumn{2}{c||}{$C a=0.4$} \\
\cline { 2 - 5 } & $\mu_{r}=1$ & $\mu_{r}=10$ & $\mu_{r}=1$ & $\mu_{r}=10$ \\
\hline $\operatorname{Re}=1$ & 0.0089 & 0.0038 & 0.0051 & 0.0038 \\
$\operatorname{Re}=10$ & 0.0089 & 0.0064 & 0.0051 & 0.0064 \\
$\operatorname{Re}=50$ & 0.0102 & 0.0178 & 0.0064 & 0.0166 \\
$\operatorname{Re}=100$ & 0.0102 & 0.0228 & 0.0064 & 0.0216 \\
\hline
\end{tabular}

The boundary conditions applied in the above configuration at the top and bottom walls of the cavity for all the velocity components are of the Dirichlet type:

$$
\begin{aligned}
& u(x=0, y=2 D)=-u(x, y=0)=U, \\
& v(x, y=2 D)=v(x, y=0)=0 .
\end{aligned}
$$

In addition, periodic boundary conditions were applied for the velocity and pressure fields in the horizontal, $x$, direction. A single Dirichlet point for the pressure field, $p=0$, was set in the corner of the computational domain. The mesh grid $h=1 / 300$ and time step $\Delta t=10^{-4}$ were utilized in all the simulations. A comparison between the present and the previously published data [40] for the drop shapes at steady state as a function of various values of operating conditions are presented in Fig. 9. An excellent agreement between the results is observed for the entire range of parameters. It can be seen that increasing the $C a$ number results in more pronounced elongation of the drop shape, which can be explained by the reduced surface tension force. In contrast, increasing the viscosity ratio leads to decreasing the deformation of the drop shape. The mass leakage calculated for all the configurations analyzed in test case 2 is summarized in Table 4 at final time $t=3$. It can be seen that the maximal value of mass lost does not exceed $0.023 \%$ of the initial drop mass.

\subsection{Test case 3 - Rosette shape}

This benchmark investigates the dynamics of the relaxation of a rosette shaped drop to a circular disc in a quiescent viscous liquid. In this configuration, the flow is driven by the surface tension forces, while the momentum of the flow is diffused by the viscosity of the fluid. Gravity is neglected. The initial configuration in this problem consists of a rosette shaped drop. The center of the drop coincides with the center of a square cavity. The initial shape of the drop is defined by

$$
X(0 \leq \theta \leq 2 \pi)=\left\{\begin{array}{l}
X=D \cdot(0.5+r \cos (\theta)), \\
Y=D \cdot(0.5+r \sin (\theta)),
\end{array}\right.
$$

where $r=0.25+0.1 \cos (n \theta)$ and $n$ is the oscillation mode. No-slip boundary conditions were applied at all the cavity walls. In addition, a single Dirichlet point for the pres- 

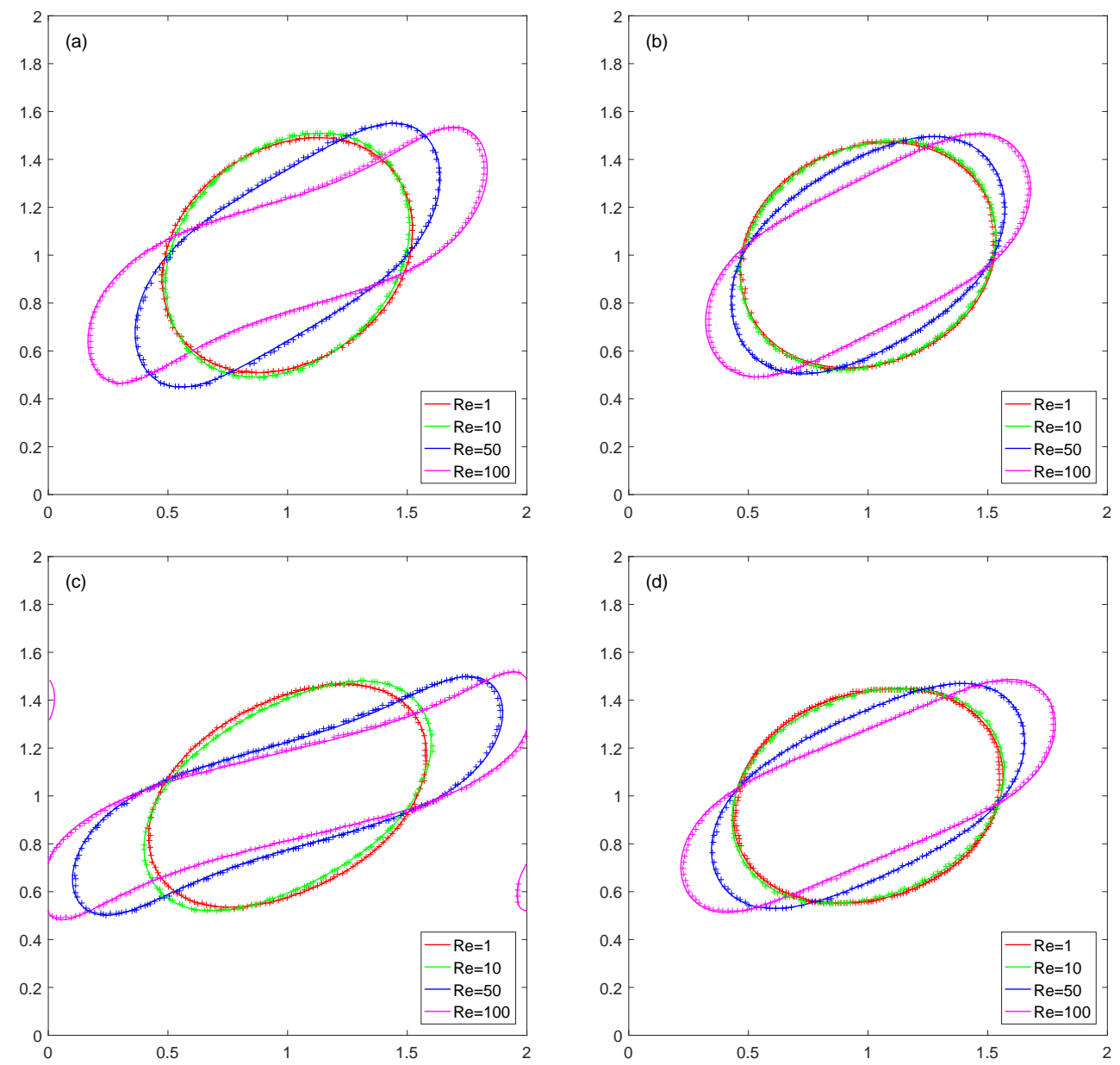

Figure 9: Drop shapes at $t=3$ : (a) $C a=0.2, \mu_{r}=1$; (b) $C a=0.2, \mu_{r}=10$; (c) $C a=0.4, \mu_{r}=1$; and (d) $C a=0.4$, $\mu_{r}=10$. Plus markers - Chinyoka et al. [40] results, lines - the present results.

sure field, $p=0$, was set in the corner of the computational domain. Following the study of Li et al. [25], the problem was studied for three different modes of $n=3,5$ and 8 as shown in Fig. 10. Since neither the characteristic velocity, nor the Reynolds and Weber number values were specified in [26], the present simulations were carried out by solving dimensional NS equations on the computational domain $[D \times D]$ with physical and geometrical parameters provided by [25], as detailed in Table 5 . The problem was solved on $h=1 / 64$ and $h=1 / 128$ grids with $\Delta t=h / 16$. Time evolution of the rosettes is visualized by presenting the rosette shapes at various time instances in the range of $0 \leq t \leq 1$, as shown in Fig. 11. Lagrangian markers determining the instantaneous rosette shapes are superimposed with the corresponding rosette shapes reported in [25]. In agreement with the Li et al. study [25], all the three modes of the rosette drop relax to a disc shape at $t=1$. However, in some instances the presently obtained rosette shapes precede the 
(a)

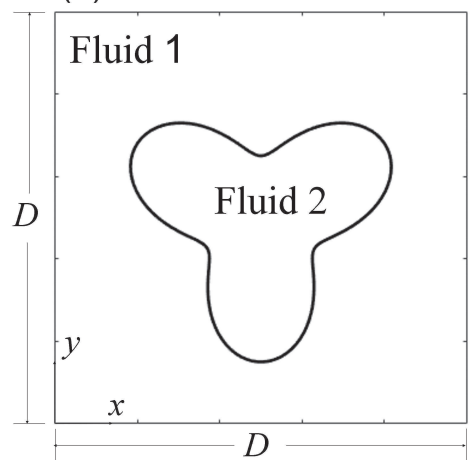

(b)

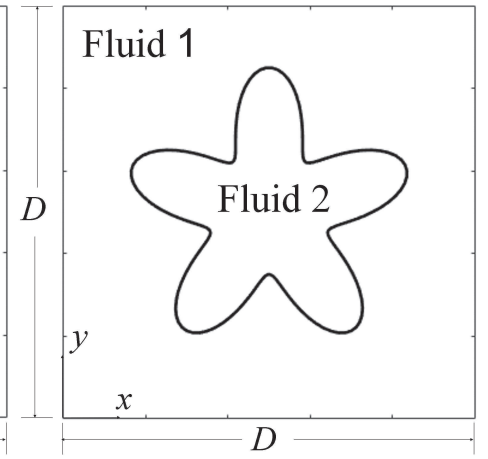

(c)

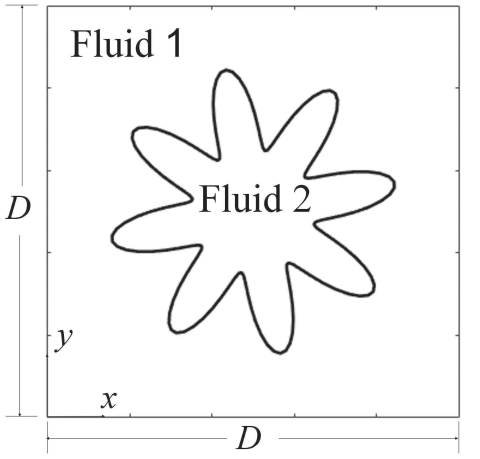

Figure 10: Initial configurations of rosette shaped drop corresponding to: (a) $n=3$ modes; (b) $n=5$ modes; (c) $n=8$ modes.

Table 5: The values of physical properties and operating conditions.

\begin{tabular}{||cccccc||}
\hline$\rho_{1}$ & $\rho_{2}$ & $\mu_{1}$ & $\mu_{2}$ & $D$ & $\sigma$ \\
\hline 1 & 1 & 0.01 & 0.01 & 1 & 130 \\
\hline
\end{tabular}

Table 6: Mass discrepancy (\%) at $t=0.5$ in test case 3.

\begin{tabular}{||cccc||}
\hline Mode & 3 & 5 & 8 \\
\hline$h=1 / 64$ & 0.4437 & 0.472 & 0.49 \\
$h=1 / 128$ & 0.2028 & 0.217 & 0.2075 \\
\hline
\end{tabular}

corresponding shapes reported in [25]. Such a discrepancy, indicating a higher rate of momentum diffusion in our simulations, can be, apparently, a consequence of differences in the distribution of Lagrangian markers at each simulation instance. Contrary to the study of Li et al. [25], in which a constant number of Lagrangian markers are used for determining the two-phase interface, the presently developed methodology utilizes a varying number of Lagrangian markers while preserving their even distribution over the interface. It can also be seen that higher modes are characterized by higher relaxation rates as a consequence of initially higher values of surface tension forces, which are proportional to the curvature of the interface separating both phases. Conservation of the area of the drop at each time step of the simulation comprises a critical criterion for verification of the developed approach, as it is dictated by the flow incompressibility. It was also verified (see Table 6) that for all simulations the maximal discrepancy in the area did not exceed $0.5 \%$ for $h=1 / 64$ and $0.21 \%$ for $h=1 / 128$.

Despite implicit representation of the surface stress forces, parasitic currents arise with maximum magnitude in the close vicinity of the interface, as shown in Fig. 12. A reason for the presence of these currents is a slight unbalance between stresses in the interfacial region. The source of the unbalanced stresses is apparently related to the truncation 

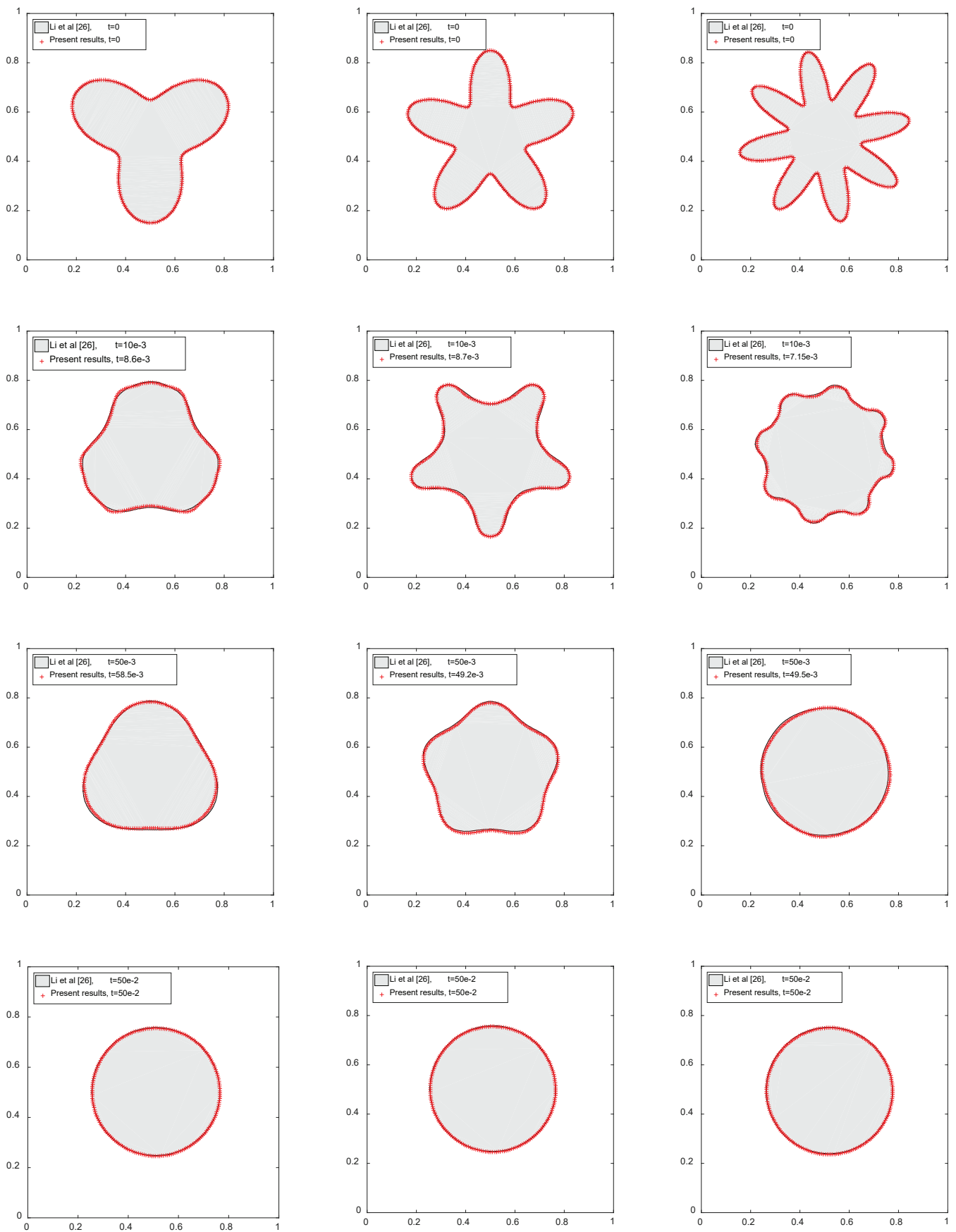

Figure 11: Verification of the time instances of a rosette drop characterized by 3,5 and 8 modes initial shape when relaxing to circular disc in quiescent liquid. 


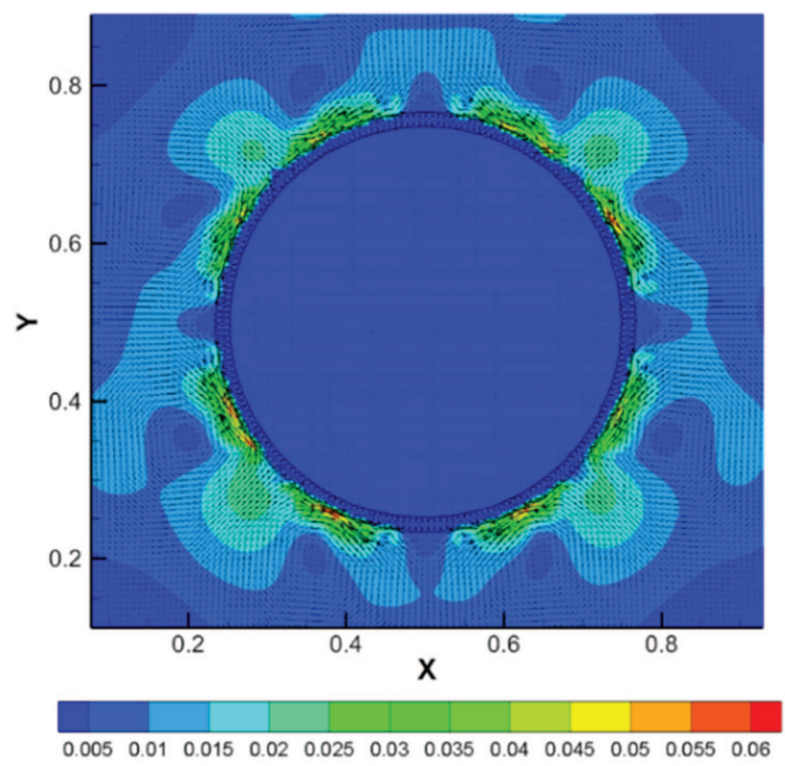

Figure 12: The magnitude of parasitic velocities observed for a drop of circular disc shape.

error associated with the second derivative of the Lagrangian coordinate (see Eq. (3.3)) when calculating the local surface tension force. Nevertheless, the maximal magnitude of the parasitic velocity does not exceed $0.6 \%$ of the characteristic velocity, which is too small to have any effect on the transient and steady state dynamics of the flow under consideration.

\subsection{Test case 4 - Rising bubble undergoing break up}

The aim of this simulation is to demonstrate that the developed methodology is also capable of simulating immiscible two-phase flows with multiple separated boundaries. For this purpose, the rise of a $2 \mathrm{D}$ gas bubble with its subsequent break up in a quiescent liquid is considered. The geometry and boundary conditions of the flow are the same as for test case 1 . The values of physical properties utilized in the present simulations are equivalent (subject to appropriate rescaling) to those used in the previous studies [43-45], as detailed in Table 7. Unfortunately, the breaking process cannot be directly captured only by the solution of the NS equations (2.1)-(2.3) with the subsequent Euler integration of

Table 7: The values of physical properties and operating conditions utilized in test case 4 .

\begin{tabular}{||ccccc||}
\hline$R e$ & $W e$ & $F r$ & $\rho_{1} / \rho_{2}$ & $\mu_{1} / \mu_{2}$ \\
\hline 120.83 & 2.6 & 1 & 40 & 83.33 \\
\hline
\end{tabular}


a. Before break up

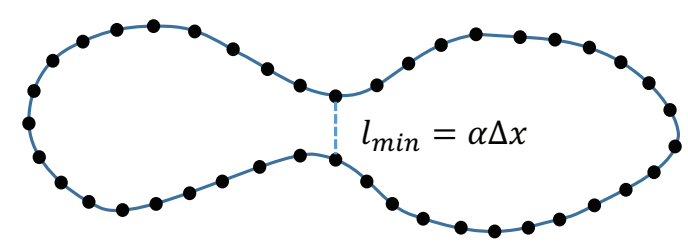

b. After break up

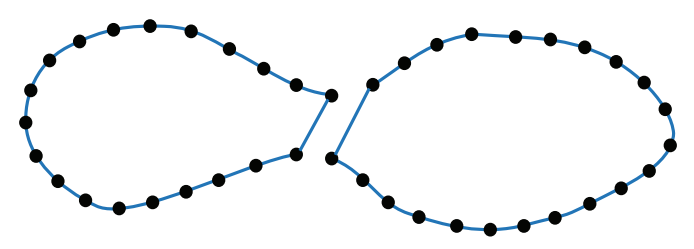

Figure 13: Schematic bubble break up modeling.

the two-phase interface governed by Eq. (3.4). Explicit modeling of the bubble break up is required. In the present study we adopt an assumption that the breaking process is followed by formation of a deformed neck, which is thinned by the dynamic local pressure. This scenario is realistic for many physical systems, for example for the microfluidic dynamics of two-phase immiscible flow in T-junctions [50-52] and is favorably applied here to simulate bubble break up in buoyant and pure shear flows. A schematic of bubble break up modeling is shown in Fig. 13. The distance between all points of the body, with the exception of a pair of points in the immediate neighborhood, is evaluated for every point of the immersed surface at each computational time step. Once the absolute minimal distance between any two points of the body $l_{\min }$ is less than a given threshold $\alpha \Delta r$ (see Fig. 13-a) the body is split into two parts (see Fig. 13-b), where each part is treated as a separate body at the next computational time. In this study, the value of $\alpha$ was set in the range of $1 \leq \alpha \leq 1.5$, which corresponds to the grid size length and to the range of influence of the presently utilized discrete Delta function, respectively. Although the above splitting procedure could result in a certain loss of mass, it was verified that for the chosen range of values the mass discrepancy of a single break up phenomenon was within the discretization error of the numerical scheme ${ }^{\ddagger}$. After the splitting, Lagrangian points of each newly formed body were redistributed to preserve an even spacing with the distance approximately equal to the size of Eulerian grid.

The time evolution of the rising initially cylindrical bubble simulated for the physical parameters listed in Table 7 is shown in Fig. 14 for $t=1.5,3,4.5,6$ instances. Mass conservation of the rising bubble was verified at all the time instances (see Table 8 ). It can be seen that the maximum mass lost does not exceed $0.15 \%$ of the initial bubble mass. The instantaneous shape of the bubble is superimposed with contours corresponding to the absolute values of the velocity vector, $|\boldsymbol{u}|$. The obtained results are in acceptable agreement with the results of Gaudlitz and Adams [43], Wang et al. [44] and Archer and Bai [45] for $t=1.5$ and $t=3$. For these time instances the symmetry of the flow relatively

‡The described break up procedure should be empirically rescaled to preserve mass if multiple break up phenomena take place. 
Table 8: Mass discrepancy (\%) calculated at every time instance in test case 4.

\begin{tabular}{||ccccc||}
\hline Time & 1.5 & 3 & 4.5 & 6 \\
\hline Mass leakage [\%] & 0.1664 & 0.2858 & 0.1141 & 0.1346 \\
\hline
\end{tabular}
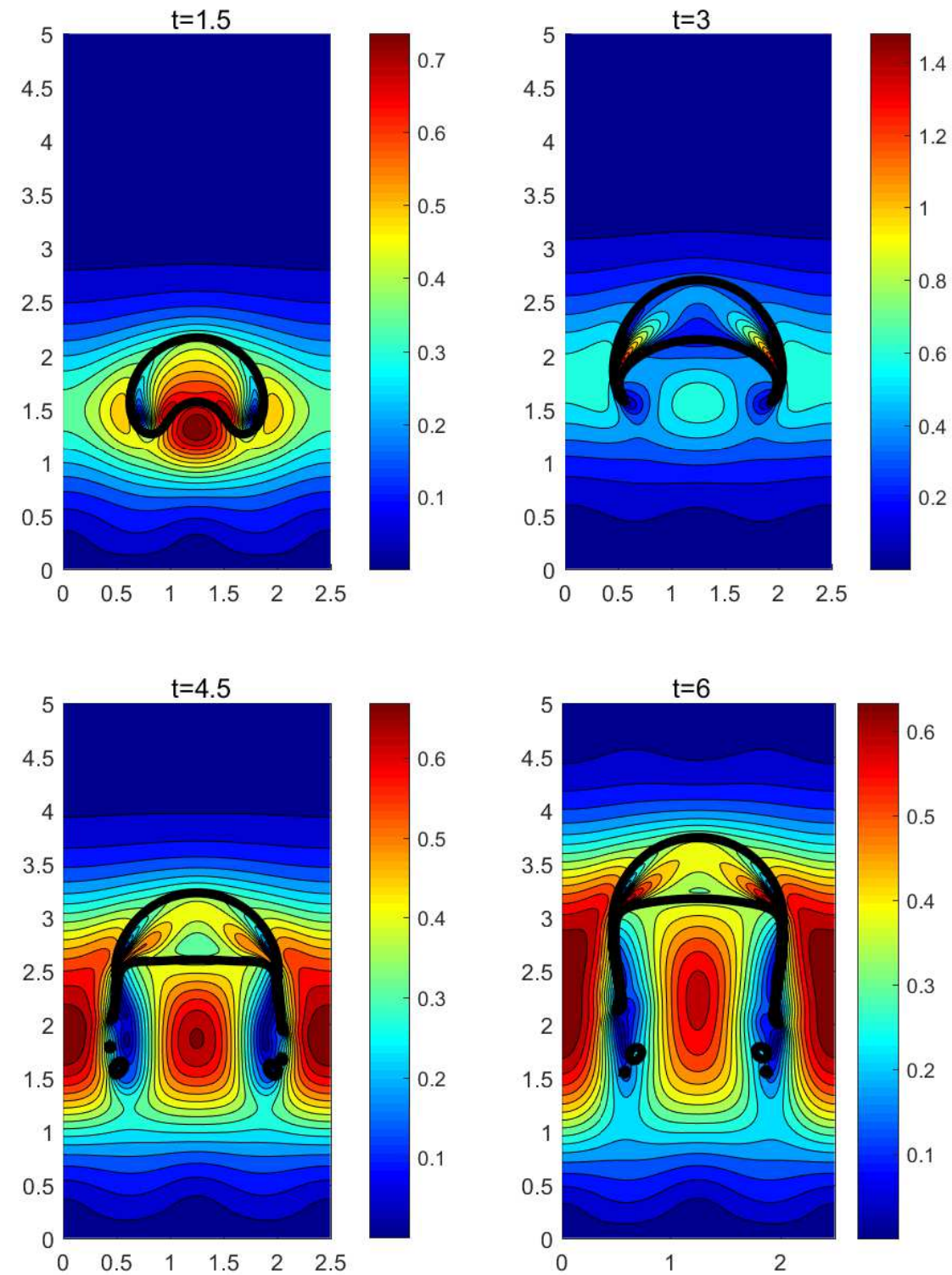

Figure 14: Time evolution of the rising initially cylindrical bubble obtained on a $320 \times 640$ grid for the value of $\alpha=1.5$ and zero initial velocity. Initial position of the bubble center was $(1.25,1.0)$. The colors correspond to absolute values of the velocity vector, $|\boldsymbol{u}|$. 
to the vertical centerline is preserved. It can be also seen that at $t=4.5$ the drop has already undergone its primary and secondary break ups followed by the slight symmetry breaking in the shapes of the major and four small pinched off bubbles. The crescent thin arms from both sides of the bubble continue to elongate even more as the bubble rises. The flow dynamics after the break up is somewhat different from that observed in the previous studies [43-45] based on the LS method. One of the reasons for the observed discrepancies could be attributed to the different grid resolutions: the present results were obtained on a $320 \times 640$ grid while all the previously reported results were obtained on a rather dense $40 \times 80$ grid. It should be noted that when such a dense grid was applied for the present methodology it provided inconclusive results, which can be seen as a drawback of the FT approach compared to its LS counterpart. On the other hand, the results obtained by the LS method can be under resolved, which can result in mass conservation violation. An additional reason for the differences in the post break up shapes of the bubbles could be due to the modeling of the break up process adopted in the present study, which can be further modified by various fine-tuning strategies to better comply with existing experimental or numerical results. Recalling that the main purpose of this simulation was to demonstrate the capability of the developed algorithm to handle multiple separated boundaries, development of such fine-tuning strategies was beyond the scope of the present study.

\section{Additional capabilities of the algorithm}

In this section we examine additional capabilities of the algorithm aimed at demonstrating that the developed methodology can favorable address both shear and buoyancydriven flows in which multiple break up phenomena take place.

We first simulate a multiple break up phenomenon for the flow configuration consisting of a circular drop under the action of pure shear flow. The drop is placed in the center of a long $10 \times 1$ channel with no-slip velocities $U \hat{i}$ and $-U \hat{i}$ at the top and the bottom walls, respectively, and periodic boundary conditions in the horizontal direction. Gravity is neglected. The values of physical parameters utilized in the simulation are given in Table 9. The time evolution of the drop shape is shown in Fig. 15. At times $70 \leq t \leq 100$ the obtained results qualitatively repeat the instability phenomena observed at the central portion of bubble [53] $]^{\S}$. The instability is preceded by elongation of the drop, which resembles the shape of a dumbbell at $t=40$, and is then followed by detachment of daughter drops via an end-pinching mechanism at $40 \leq t \leq 70$. It is remarkable that while spreading over the channel the slope of the elongated drop and of a series of its daughter drops further created as a result of the drop break up asymptotically decreases to approximately zero. We next simulate a multiple break up phenomenon by analyzing the configuration corresponding to the rising air bubble in water. The simulation was performed for the physical parameters listed in Table 10. Density and dynamic viscosity ratios were chosen

\footnotetext{
$\S$ The values of $R e$ and $W e$ were multiplied by a factor of 8 to fit the scaling of the present study.
} 

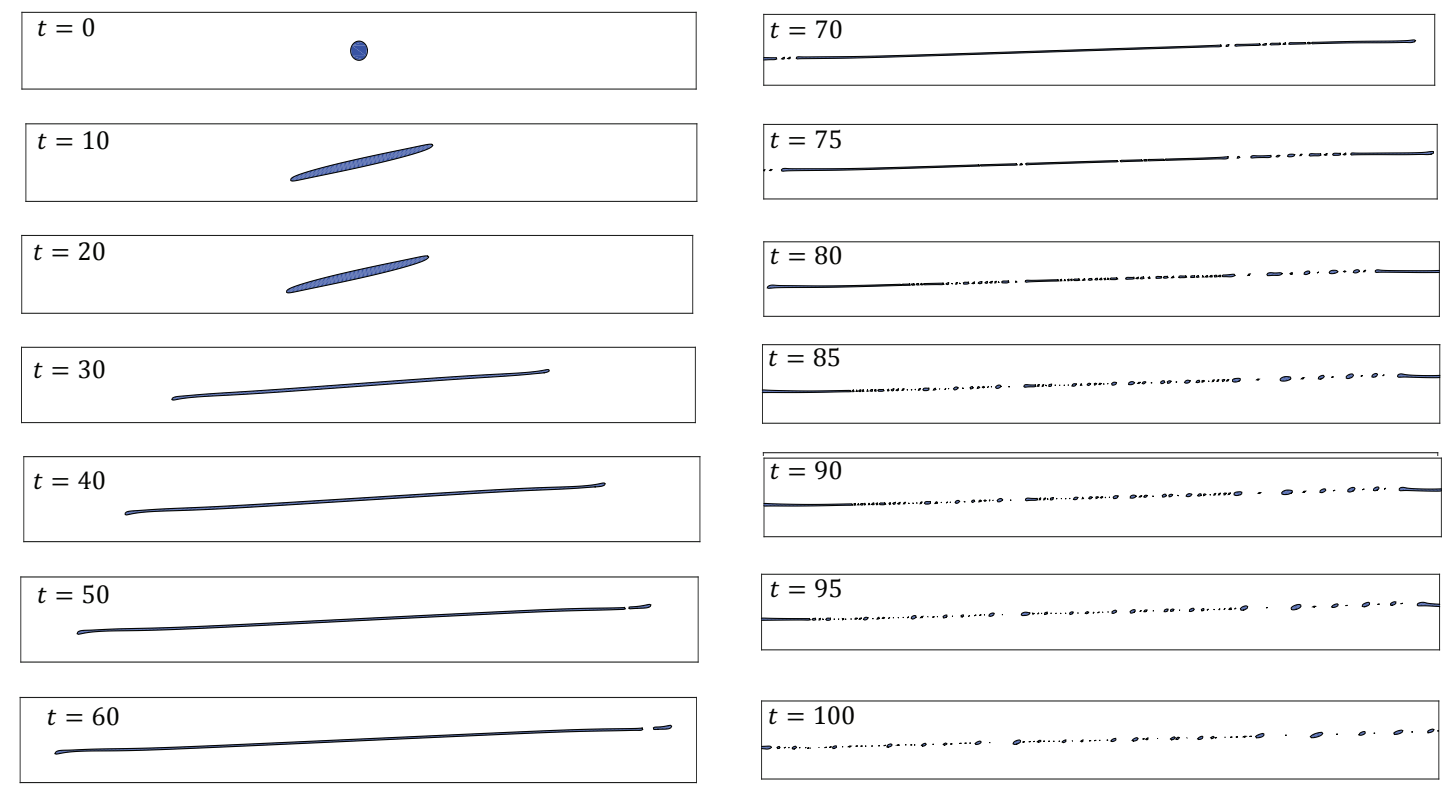

Figure 15: Interface evolution of circular drop under pure shear flow undergoing multiple break up phenomena, $\operatorname{Re}=1, W e=7.2,10 \times 1$ channel, obtained on a $50 \times 500$ grid.

Table 9: The values of physical properties and operating conditions used for 10:1 pure shear flow.

\begin{tabular}{||ccccc||}
\hline$R e$ & $W e$ & $F r$ & $\rho_{1} / \rho_{2}$ & $\mu_{1} / \mu_{2}$ \\
\hline 1 & 7.2 & 1 & 1 & 1 \\
\hline
\end{tabular}

Table 10: The values of physical properties and operating conditions utilized for the configuration corresponding to air bubble rising in water.

\begin{tabular}{||ccccc||}
\hline$R e$ & $W e$ & $F r$ & $\rho_{1} / \rho_{2}$ & $\mu_{1} / \mu_{2}$ \\
\hline 300 & 2.6 & 1 & 1000 & 185 \\
\hline
\end{tabular}

to fit air-water two-phase flow. The relatively high value of We number was taken to diminish the surface tension effects and to ensure that the break up phenomenon takes place before the bubble reaches its terminal velocity. The time evolution of a rising bubble for a number of time instances $t=0.5,1,1.5,2,2.5,3$ is presented in Fig. 16. At the beginning of the motion the bubble undergoes deformation stages similar to those observed for test case 4 . The crescent shape of the air bubble at $t=0.5$ is transformed to the shape of a dumbbell followed by further twisting and thinning at its ends and finally leading to the flow instability and multiple break up phenomenon at $t=3$. 

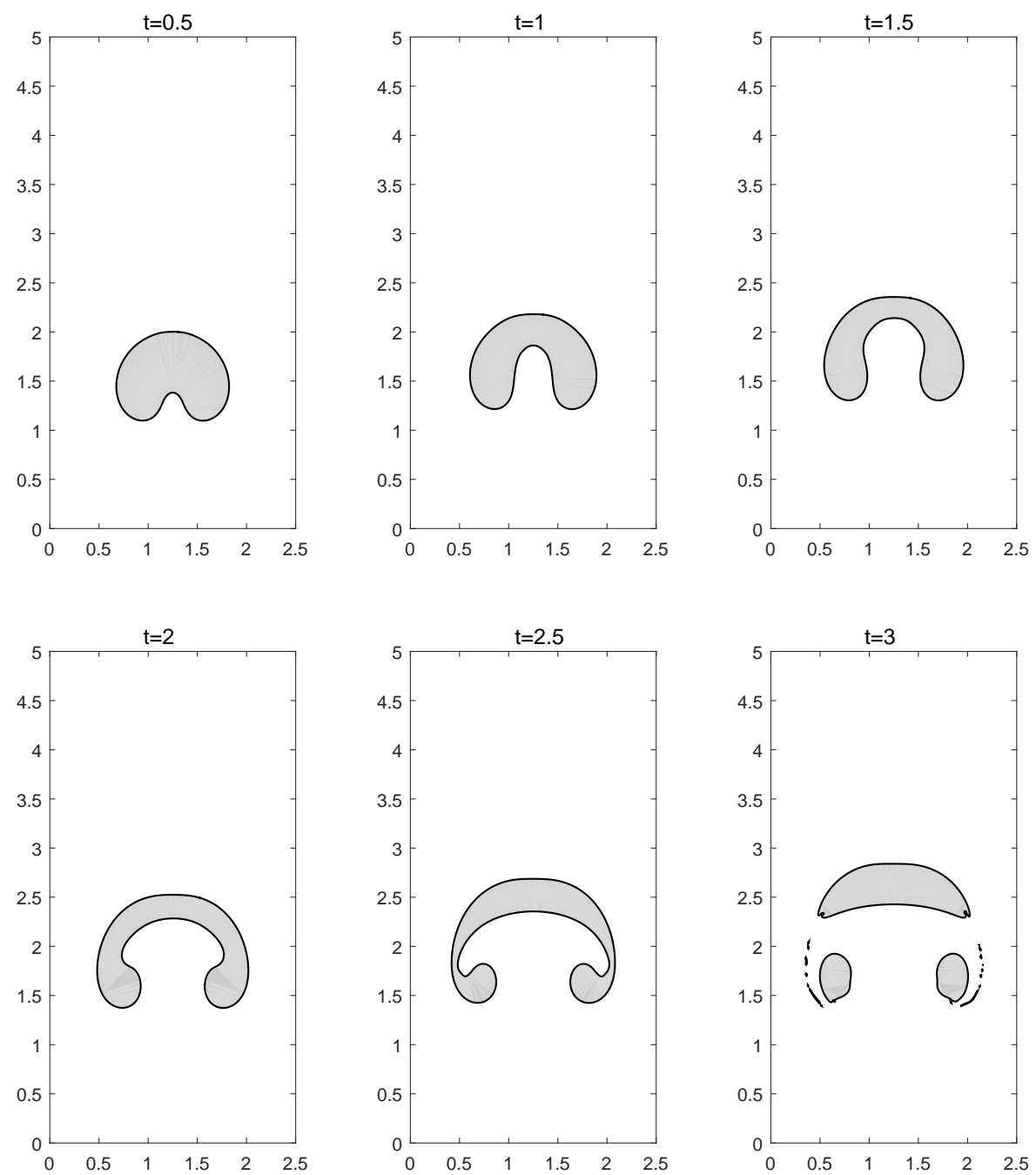

Figure 16: Interface evolution of circular air bubble rising in water. The simulations were obtained for $R e=300$, $W e=2.6, F r=1$ on a $320 \times 640$ grid.

\section{Conclusions}

In the framework of the present study, a novel methodology was developed extending the IB method based on the distributed Lagrange multiplier approach to configurations relevant to two-phase immiscible flows. The capabilities of the developed method were demonstrated by utilizing the fully pressure-velocity coupled direct solver (FPCD) [34] as a computational platform. The idea underlying the developed approach is that the initial system of fully pressure-velocity coupled NS equations is extended by including addi- 
tional relationships implicitly linking between the Euler flow fields and the distributed Lagrange multipliers, enforcing kinematic constraints of the impermeability of both fluids on the interface separating the two phases.

The fully coupled formulation enables accurate mass conservation of both phases without the need for any additional correction procedure, which is of significant computational advantage. The developed method was extensively verified for four typical twophase flows dominated by buoyancy, shear stress and surface tension forces. For all cases, a satisfactory quantitative agreement with independent numerical results available in the literature was achieved, verifying the developed methodology. The grid independence of the obtained results was also established. The capability of the developed method to handle two-phase flows with high values of density and viscosity ratios and low values of surface tension was demonstrated. A complex multiple break up phenomenon was favorable modelled for a number of representative shear and buoyancy-driven flows.

Our approach can be applied for the simulation of 2D and axi-symmetric flows, as well as for the analysis of 3D flows with periodic boundary conditions in the third, span wise, direction. The focus of our future work will be: first, introducing modeling for merging of multiple surfaces and second, extension of this approach to the simulation of general 3D flows. The first goal will make it possible to simulate mixing flow phenomena typical for classical dam break flow, for example, and will upgrade the overall flexibility of the developed approach bringing it closer to functionalities of VOF and LS methods. The second goal will be achieved by utilizing segregated pressure-velocity coupling, such as the SIMPLE algorithm (or its derivatives) combined with the Schur complement method, as detailed in our recent work [54] addressing natural convection flow in the presence of immersed bodies of an arbitrary geometry.

\section{References}

[1] S. B. Pope, Turbulent flows, IOP Publishing, 2001.

[2] D. A. Drew, "Mathematical modeling of two-phase flow" Annual review of fluid mechanics, vol. 15, pp. 261-291, 1983.

[3] K. A. Rejniak, H. J. Kliman and L. J. Fauci, "A computational model of the mechanics of growth of the villous trophoblast bilayer" Bulletin of mathematical biology, vol. 66, pp. 199$232,2004$.

[4] K. A. Rejniak, "An immersed boundary framework for modelling the growth of individual cells: an application to the early tumour development," Journal of theoretical biology, vol. 247, pp. 186-204, 2007.

[5] A.-L. Dessimoz, L. Cavin, A. Renken and L. Kiwi-Minsker, "Liquid-liquid two-phase flow patterns and mass transfer characteristics in rectangular glass microreactors," Chemical Engineering Science, vol. 63, pp. 4035-4044, 2008.

[6] E. Maitre, "Review of numerical methods for free interfaces," Ecole Thematique, Les Houches, 2006.

[7] G. Tryggvason, B. Bunner, A. Esmaeeli, D. Juric, N. Al-Rawahi, W. Tauber, J. Han, S. Nas and Y. J. Jan, "A Front tracking method for the computations of multiphase flow," Journal of computational physics, vol. 169, pp. 708-759, 2001. 
[8] G. Tryggvason, R. Scardovelli and S. Zaleski, Direct numerical simulations of gas-liquid multiphase flows, Cambridge University Press, 2011.

[9] C. S. Peskin, "The immersed boundary method," Acta numerica, vol. 11, pp. 479-517, 2002.

[10] C. S. Peskin, "Numerical analysis of blood flow in the heart," Journal of computational physics, vol. 25.3, pp. 220-252, 1977.

[11] A. M. Roma, C. S. Peskin and M. J. Berger, "An adaptive version of the immersed boundary method," Journal of computational physics, vol. 153.2, pp. 509-534, 1999.

[12] Y. Li, A. Yun, D. Lee, J. Shin, D. Jeong and J. Kim, "Three-dimensional volume-conserving immersed boundary model for two-phase fluid flows," computation methods application mechanical engineer, vol. 257, pp. 36-46, 2013.

[13] C. W. Hirt and B. D. Nichols, "Volume of fluid (VOF) method for the dynamics of free boundary," Journal of computational physics, vol. 39.1, pp. 201-225, 1981.

[14] N. Balczar, L. Jofre, O. Lehmkuhl, J. Castro and J. Rigola, "A finite-volume/level-set method for simulating two-phase flows on unstructured grids," International journal of multiphase flow, pp. 55-72, 2014.

[15] D. Jacqmin, "Calculation of two-phase Navier-Stokes flows using Phase-Field modeling," Journal of computational phisics, vol. 155, pp. 96-127, 1999.

[16] J. Kim, "Phase field models for multi-component fluid flows," Communications in Computational Physics, vol. 12.3, pp. 613-661, 2012.

[17] J. Mohd-Yusof, "Combined immersed-boundary/b-spline methods for simulations of flow in complex geometries, in: Annual Research Briefs," Center for Turbulence Research, 1997.

[18] E. Fadlun, R. Verzicco, P. Orlandi and J. Mohd-Yusof, "Combined immersed-boundary finite-difference methods for three-dimensional complex flow simulations, "Journal of computational physics, vol. 161, no. 1, pp. 35-60, 2000.

[19] V. Rutka and Z. Li, "An explicit jump immersed interface method for two-phase NavierStokes equations with interfaces," Computer Methods in Applied Mechanics and Engineering, vol. 197, pp. 2317-2328, 2008.

[20] S. Patankar, Numerical Heat Transfer and Fluid Flow, CRC press, 1980.

[21] L. Lee and R. J. Leveque, "An immersed interface method for incompressible Navier-Stokes equations", SIAM Journal on Scientific Computing, vol. 25, pp. 832-856, 2003.

[22] M. N. Linnick and H. F. Fasel, "A high-order immersed interface method for simulating unsteady incompressible flows on irregular domains," Journal of Computational Physics, vol. 204, pp. 157-192, 2005.

[23] T. Kempe and F. Jochen, "An improved immersed boundary method with direct forcing for the simulation of particle laden flows," Journal of Computational Physics, vol. 231, pp. 3663-3684, 2012.

[24] T. Kempe, M. Lennartz, S. Schwarz and J. Frhlich, "Imposing the free-slip condition with a continuous forcing immersed boundary method," Journal of Computational Physics, vol. 282, pp. 183-209, 2015.

[25] Y. Li, E. Jung, W. Lee, H. G. Lee and J. Kim, "Volume preserving immersed boundary methods for twophase fluid flows," International Journal for Numerical Methods in Fluids, vol. 69.4, pp. 842-858, 2012.

[26] Y. Bao, A. Donev, B. E. Griffith, D. M. McQueen and C. S. Peskin, "An Immersed Boundary method with divergence-free velocity interpolation and force spreading," Journal of Computational Physics, vol. 347, pp. 183-206, 2017.

[27] R. Glowinski, P. Tsorng-Whay and J. Periaux, "Distributed Lagrange multiplier methods for incompressible viscous flow around moving rigid bodies," Computer methods in applied 
mechanics and engineering, vol. 151, pp. 181-194, 1998.

[28] R. Glowinski, T. Pan, T. Hesla, D. Joseph and J. Periaux, "A fictitious domain approach to the direct numerical simulation of incompressible viscous flow past moving rigid bodies: application to particulate flow," Journal of Computational Physics, vol. 169, pp. 363-426, 2001.

[29] Z. Yu, N. Phan-Thien and Y. Fan, "Viscoelastic mobility problem of a system of particles," Journal of non-newtonian fluid mechanics, vol. 104, pp. 87-124, 2002.

[30] Z. Yu, N. Phan-Thien and R. Tanner, "Dynamic simulation of sphere motion in a vertical tube," Journal of Fluid Mechanics, vol. 518, pp. 61-93, 2004.

[31] Z. Yu, "A DLM/FD method for fluid/flexible-body interactions," Journal of computational physics, vol. 207, pp. 1-27, 2005.

[32] K. Taira and T. Colonius, "The immersed boundary method: a projection approach," Journal of Computational Physics, vol. 225, pp. 2118-2137, 2007.

[33] Y. Feldman and Y. Gulberg, "An extension of the immersed boundary method based on the distributed Lagrange multiplier approach," Journal of Computational Physics, vol. 322, pp. 248-266, 2016.

[34] Y. Feldman and A. Y. Gelfgat, "On pressure-velocity coupled time-integration of incompressible Navier-Stokes equations using direct inversion of Stokes operator or accelerated multigrid technique," Computers and Structures, vol. 87, no. 11, pp. 710-720, 2009.

[35] S. J. Shin, W.-X. Huang and H. J. Sung, "Assessment of regularized delta functions and feedback forcing schemes for an immersed boundary method," International Journal for Numerical Methods in Fluids, vol. 58.3, pp. 263-286, 2008.

[36] P. R. Amestoy, I. S. Duff, J.-Y. L'Excellent and J. Koster, "A fully asynchronous multifrontal solver using distributed dynamic scheduling," SIAM Journal on Matrix Analysis and Applications, vol. 23.1, pp. 15-41, 2001.

[37] P. R. Amestoy, A. Guermouche, J.-Y. LExcellent and S. Pralet, "Hybrid scheduling for the parallel solution of linear systems," Parallel computing, vol. 32.2, pp. 136-156, 2006.

[38] S. Hysing, S. Turek, D. Kuzmin, N. Parolini, E. Burman, S. Ganesan and L. Tobiska, "Quantitative benchmark computations of twodimensional bubble dynamics, "International Journal for Numerical Methods in Fluids, vol. 60.11, pp. 1259-1288, 2009.

[39] J. Klostermann, K. Schaake and R. Schwarze, "Numerical simulation of a single rising bubble by VOF with surface compression," International Journal for Numerical Methods in Fluids, vol. 71.8, pp. 960-982, 2013.

[40] T. Chinyoka, Y. Renardy, M. Renardy and D. Khismatullin, "Two-dimensional study of drop deformation under simple shear for Oldroyd-B liquids," Journal of Non-Newtonian Fluid Mechanics, vol. 130, pp. 45-56, 2005.

[41] K. S. Sheth and C. Pozrikidis, "Effect of inertia on the deformation of liquid drops in simple shear flow," Computers and Fluids, vol. 24.2, pp. 101-119, 1995.

[42] F. Ilinca and J.-F. Htu, "Immersed Boundary Solution of Natural Convection in a Square Cavity with an Enclosed Rosette-Shaped Hot Cylinder," Numerical Heat Transfer, Part A: Applications, vol. 65.12, pp. 1154-1175, 2014.

[43] D. Gaudlitz and N. A. Adams, "On improving mass-conservation properties of the hybrid particle-level-set method, " Computers \& Fluids, vol. 37, pp. 1320-1331, 2008.

[44] Z. Wang, J. Yang and F. Stern, "An improved particle correction procedure for the particle level set method," Journal of Computational Physics, vol. 228, pp. 5819-5837, 2009.

[45] P. J. Archer and W. Bai, "A new non-overlapping concept to improve the Hybrid Particle Level Set method in multi-phase fluid flows," Journal of Computational Physics, vol. 282, 
pp. 317-333, 2015.

[46] R. Clift, J. R. Grace and M. E. Weber, Bubbles, Drops, and Particles, New York: Acadenic press, 1978.

[47] S.-R. Hysing, "Numerical simulation of immersed fluids with FEM level set techniques," Techn. Univ, 2007.

[48] M. Kronbichler and G. Kreiss, "A hybrid level-set-phase-field method for two-phase flow with contact lines," University of Uppsala, 2011.

[49] H. Wadell, "Sphericity and roundness of rock particles," The Journal of Geology, vol. 41, no. 3, pp. 310-331, 1933.

[50] T. Fu, Y. Ma and H. Z. Li, "Hydrodynamic feedback on bubble breakup at a T-junction within an asymmetric loop," AIChE Journal, vol. 60, no. 5, pp. 1920-1929, 2014.

[51] Y. Song, P. Manneville and C. N. Baroud, "Local interactions and the global organization of a two-phase flow in a branching tree," Physical review letters, vol. 105, no. 13, p. 134501, 2010.

[52] A. Leshansky and L. Pismen, "Breakup of drops in a microfluidic T junction," Physics of Fluids, vol. 21, no. 2, p. 023303, 2009.

[53] J. Li, Y. Y. Renardy and M. Renardy, "Numerical simulation of breakup of a viscous drop in simple shear flow through a volume-of-fluid method," Physics of Fluids, vol. 12, no. 2, pp. 269-282, 2000.

[54] Y. Feldman, "Semi-implicit direct forcing immersed boundary method for incompressible viscous thermal flow problems: a Schur complement approach," Int. J. Heat Mass Transfer (2018), https://doi.org/10.1016/j.ijheatmasstransfer.2018.06.099 